Article

\title{
Comparison of Two Doses of Elemental Iron in the Treatment of Latent Iron Deficiency: Efficacy, Side Effects and Blinding Capabilities
}

\author{
Alecia J. Leonard ${ }^{1}$, Kerry A. Chalmers ${ }^{2, *}$, Clare E. Collins ${ }^{1}$ and Amanda J. Patterson ${ }^{1}$ \\ 1 Priority Research Centre in Physical Activity and Nutrition and School of Health Sciences, \\ Faculty of Health and Medicine, University of Newcastle, Callaghan, NSW 2308, Australia; \\ E-Mails: Alecia.Leonard@uon.ed.au (A.J.L.); Clare.Collins@newcastle.edu.au (C.E.C.); \\ Amanda.Patterson@newcastle.edu.au (A.J.P.) \\ 2 School of Psychology, Faculty of Science and IT, University of Newcastle, Callaghan, \\ NSW 2308, Australia
}

* Author to whom correspondence should be addressed; E-Mail: Kerry.Chalmers@newcastle.edu.au; Tel.: +61-2-4921-5757; Fax: +61-2-4921-6906.

Received: 9 January 2014; in revised form: 24 March 2014 / Accepted: 26 March 2014 / Published: 4 April 2014

\begin{abstract}
Adherence to iron supplementation can be compromised due to side effects, and these limit blinding in studies of iron deficiency. No studies have reported an efficacious iron dose that allows participants to remain blinded. This pilot study aimed to determine a ferrous sulfate dose that improves iron stores, while minimising side effects and enabling blinding. A double-blinded RCT was conducted in 32 women (18-35 years): 24 with latent iron deficiency (serum ferritin $<20 \mu \mathrm{g} / \mathrm{L}$ ) and 8 iron sufficient controls. Participants with latent iron deficiency were randomised to $60 \mathrm{mg}$ or $80 \mathrm{mg}$ elemental iron or to placebo, for 16 weeks. The iron sufficient control group took placebo. Treatment groups (60 $\mathrm{mg} n=7$ and $80 \mathrm{mg} n=6$ ) had significantly higher ferritin change scores than placebo groups (iron deficient $n=5$ and iron sufficient $n=6), F(1,23)=8.46, p \leq 0.01$. Of the 24 who completed the trial, 10 participants $(77 \%)$ on iron reported side effects, compared with $5(45 \%)$ on placebo, but there were no differences in side effects $(p=0.29)$, or compliance $(p=0.60)$ between iron groups. Nine (69\%) participants on iron, and $11(56 \%)$ on placebo correctly guessed their treatment allocation. Both iron doses were equally effective in normalising ferritin levels. Although reported side-effects were similar for both groups, a majority of participants correctly guessed their treatment group.
\end{abstract}


Keywords: ferrous sulfate; side effects; women; serum ferritin

\section{Introduction}

Young women are at high risk of iron deficiency secondary to menstruation and childbirth [1]. The nutritional disorder affects one in five young women in Australia [2] and is associated with poorer general health and wellbeing and high levels of fatigue $[3,4]$. It is imperative that iron deficiency is effectively managed to prevent progression to anaemia. Increased dietary iron intake, iron fortification and iron supplementation are used to improve iron status [5]. Clinical practice guidelines for the management of iron deficiency have been developed in the United States [6], the United Kingdom [7] and in Australia [8,9]. These all recommend the use of dried ferrous sulfate which contains approximately $33 \%$ elemental iron. Clinical practice guidelines recommend a daily dose of $80-105 \mathrm{mg}$ of elemental iron for treatment of iron deficiency anaemia in adults [10]. A systematic review conducted in 2011 assessed the effects of intermittent oral iron supplementation on anaemia in menstruating women, compared with no intervention, a placebo or daily supplementation [11]. This study found weekly supplementation with 60 to $120 \mathrm{mg}$ elemental iron was effective in improving haematological markers [11]. Treatment of latent iron deficiency and the impact of using lower dose iron treatment on iron status are not articulated within current literature and iron treatment guidelines.

Ideally, supplementation should achieve maximal absorption with minimal side effects [12]. Oral iron has been associated with gastrointestinal side effects such as nausea, constipation and darkening of stools which can decrease compliance $[8,13]$. Such side effects can compromise blinding within trials. Lower dose iron supplements have fewer side effects $[10,14]$ yet the effect of varying the dosage of iron on iron status [15-17] has rarely been studied, with no studies conducted in non-pregnant young women. Whether lower doses are absorbed as efficiently as higher doses in non-pregnant young women remains unknown [18]. Therefore, the current study aims to determine the efficacy of two different doses of iron supplementation in improving iron status whilst maintaining blinding to treatment groups.

\section{Experimental Section}

Testing was conducted at the University of Newcastle, Callaghan Campus in NSW, Australia between April 2010 and April 2013. Women aged 18-35 years were recruited via flyers and promotion in lectures within the University. Recruitment also included flyers at the Technical Education (TAFE) College, accessing the volunteer register at Hunter Medical Research Institute and word-of-mouth. All interested individuals were screened for eligibility against inclusion criteria using an author designed questionnaire (refer to supplementary material). The inclusion criteria were: female, 18-35 years; BMI $18-30 \mathrm{~kg} / \mathrm{m}^{2}$; English as primary language; not iron deficient within the last 12 months; not currently taking iron supplementation (those who had been on a standard multivitamin, containing minimal or no iron, were eligible to participate and asked to cease the supplement); no chronic medical condition; not taking medication that could potentially interfere with results; ability to provide blood samples for biomarkers of iron status; not having donated blood within the last three months and will not donate 
blood during the trial; not pregnant, or planning a pregnancy within the following 4 months; available to participate in intervention for 4 months. Those eligible were provided with an information statement and informed consent was obtained prior to the commencement of the study.

\subsection{Participants}

Thirty two women were included in the intervention. As shown in Figure 1, eight participants were included in the iron sufficient control group and were provided placebo capsules, and 24 iron deficient participants were randomised to either placebo, or treatment (60 mg or $80 \mathrm{mg}$ iron).

Figure 1. Study recruitment and randomisation flow chart.

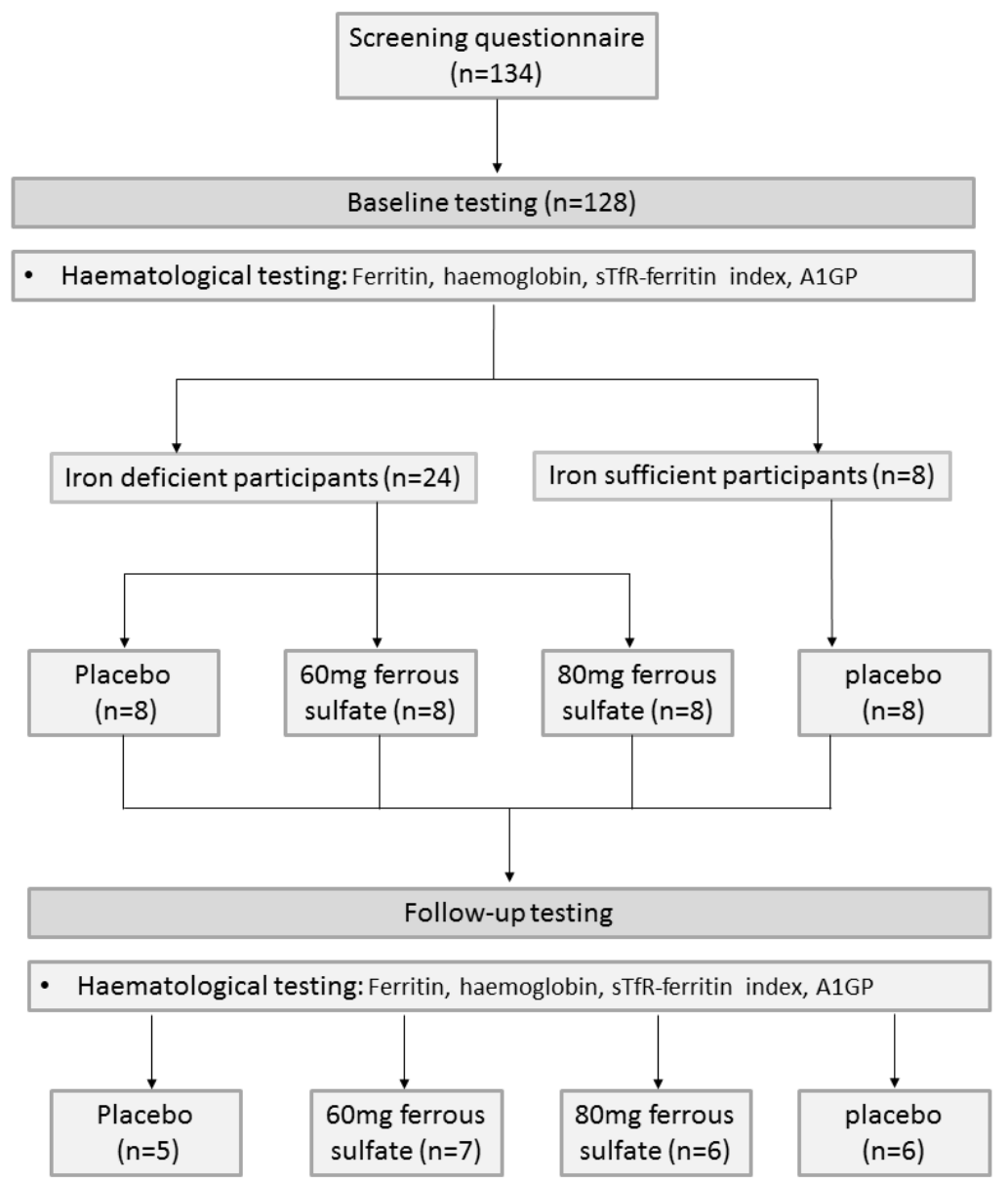

Abbreviations: sTfR; soluble transferrin receptor, A1GP; Alpha-1-Glycoprotein.

\subsection{Haematological Testing}

Serum ferritin, haemoglobin and soluble transferrin receptor (sTfR) were used as biomarkers of iron status, and alpha-1glycoprotein (A1GP) was used as a marker of inflammation. A1GP is slower to rise, but remains at a high concentration longer than $\mathrm{C}$-reactive protein (CRP), so is a better indicator of chronic sub-clinical infection than CRP, and may better reflect changes in the concentration of ferritin during infections [1]. Blood tests were performed by Hunter Area Pathology Service, accredited by the National Association of Testing Authorities Australia, using standard techniques. The timing of the blood testing was not restricted in order to optimise recruitment and compliance [19]. Results of the 
blood tests were sent directly to the research team at the University, and participants remained blinded to blood test results until the completion of the trial. Iron deficiency was defined as having ferritin $<20 \mu \mathrm{g} / \mathrm{L}$ [20] and all other markers within reference ranges (haemoglobin 115-165 g/L [20], soluble transferrin receptor $0.9-2.30 \mathrm{mg} / \mathrm{L}$ [21,22], A1GP 0.51-1.17 g/L [23]). sTfR reflects the number of iron receptors expressed on cell membranes and is raised once tissue iron starts to become limited [24]. It should theoretically represent a definitive marker of latent iron deficiency [25]. Participants with haemoglobin results below the reference range were excluded from the intervention and were immediately referred to their General Practitioner. At completion of the trial, all women were given copies of their blood test results for communication with their General Practitioner.

\subsection{Pilot Testing of Supplementation}

Figure 1 summarises group allocation and progression through the trial. Young women found to be iron deficient at baseline were randomly assigned to one of two different doses (60 $\mathrm{mg}$ or $80 \mathrm{mg}$ ) of ferrous sulfate or placebo for 16 weeks. Ferrous sulfate was used as it is the most common type of elemental iron used to treat iron deficiency; two different doses were used to determine the most effective dose in improving iron status with the fewest side-effects. The doses $60 \mathrm{mg}$ and $80 \mathrm{mg}$ have been associated with fewer side-effects than the doses recommended in the National Guidelines [10,14]. The duration of supplementation was chosen based upon the correction of iron deficiency anaemia taking between 2 and 4 months [10]. The first eight iron sufficient participants were invited into the intervention as a control group. A single blinding approach was used with the control group who were all provided with placebo capsules, which contained Lactose. Subsequent iron sufficient participants exited the study following baseline testing. Participants were not informed of their treatment or iron status until trial completion. All participants were contacted on a four weekly basis to report any potential side-effects, using a specifically designed questionnaire. It was explained to participants that any remaining capsules would be counted following the intervention to increase compliance. In addition, participants were provided with a "Tips and Reminders" sheet for taking capsules. This included the following information: Take one capsule per day; Leave your container of capsules next to your tooth brush; Keep one or two capsules in the small container provided and leave this in your handbag in case you forget to take it in the morning and then remember part way through the day; Use the calendar to cross off each day once you have taken your capsule. This will help you to keep a track of how regularly you are remembering to take them; Take the capsule two hours apart from any other regular medication (except the oral contraceptive pill which can be taken at the same time); Do not take two capsules on the same day to compensate for missing your capsule the previous day-Take only one capsule per day; Return any left-over capsules in your container when you return for your follow up testing; Please take note of your compliance with the treatment regimen and any side effects you may have experienced and report this to the research team when you are contacted by phone every four weeks. Immediately following the 16 week intervention participants were asked to guess which treatment protocol they thought they had been allocated to. Participants had repeat blood tests after 16 weeks. 


\subsection{Capsules and Randomisation}

Compounding chemists were contracted to provide the iron and placebo supplements and used Random Allocation Software to allocate treatments to participant Identification Numbers [26]. The active and placebo supplements were identical in appearance and were packaged in identical containers. The researchers and participants remained blinded to the treatment protocol and the randomisation code was held by a third party researcher only to be broken once the final results were collected. The study protocol was approved by the University of Newcastle Human Research Ethics Committee.

\subsection{Statistical Analysis}

STATA-IC 11 statistical analysis software was used with an alpha level of 0.05 set for statistical significance. Kruskal-Wallis rank test was used to analyse the effect of group on iron markers at baseline and follow-up and the difference in ferritin change score between oral contraceptive pill users and non-users on iron treatment. One-way analysis of variance (ANOVA) was used to examine the difference in iron marker change scores between treatment (60 mg iron and $80 \mathrm{mg}$ iron) and no treatment groups (control and placebo). Fischer's exact test was used to examine the frequency of reported side effects and the frequency of correct treatment guesses between treatment and no treatment groups.

\section{Results}

\subsection{Participants}

Twenty-four (75\%) participants (mean age \pm SD $25.6 \pm 4.1$ years) completed the intervention (60 mg iron $n=7,80 \mathrm{mg}$ iron $n=6$, placebo $n=5$, control $n=6$ ). Reasons given for withdrawing from the study were unrelated illness $(n=3)$ or being too busy $(n=3)$. Two participants gave no reason. Participant demographics are shown in Table 1. Participants were primarily Australian, had a mean BMI of $21.2 \mathrm{~kg} / \mathrm{m}^{2}$ and $48 \%$ used an oral contraceptive pill (OCP).

Table 1. Participant demographics $(n=24)$.

\begin{tabular}{ccccc}
\hline & 60 $\mathbf{~ m g}$ iron & $\mathbf{8 0 ~} \mathbf{~ m g}$ iron & Placebo & Control \\
\hline Age $($ years $)$ & $27.9 \pm 5.1$ & $24.5 \pm 3.4$ & $24.8 \pm 3.8$ & $24.7 \pm 3.7$ \\
BMI $\left(\mathrm{kg} / \mathrm{m}^{2}\right)$ & $20.8 \pm 1.7$ & $21.7 \pm 1.2$ & $21.9 \pm 2.4$ & $20.6 \pm 2.0$ \\
Origin & & & & \\
Australia & 4 & 3 & 5 & 2 \\
Asia & 1 & 0 & 0 & 1 \\
Canada & 1 & 0 & 0 & 0 \\
United Kingdom & 0 & 1 & 0 & 0 \\
OCP use (total) & 3 & 3 & 4 & 2 \\
\hline
\end{tabular}

Note: BMI: Body mass index, Age and BMI data is provided as mean $\pm \mathrm{SD}$. 


\subsection{Iron Status}

Ferritin, haemoglobin, and sTfR levels at baseline and follow-up, together with change scores for each group (60 mg iron, $80 \mathrm{mg}$ iron, iron deficient placebo, iron sufficient control) are presented in Table 2. The A1GP was normal in all participants and was unchanged following the intervention.

Table 2. Mean $( \pm$ SEM) haematological markers of iron status at baseline, follow-up and change scores by treatment group.

\begin{tabular}{ccccc}
\hline Iron marker & 60 mg iron & $\mathbf{8 0 ~ m g}$ iron & Placebo & Control \\
\hline \multicolumn{5}{c}{ Ferritin $(\mu \mathrm{g} / \mathrm{L})$} \\
Baseline & $11.1 \pm 1.9$ & $10.5 \pm 1.7$ & $13.5 \pm 2.1$ & $30.4 \pm 2.9$ \\
Follow-up & $34.4 \pm 10.2$ & $30.7 \pm 7.0$ & $15.1 \pm 1.8$ & $31.9 \pm 5.0$ \\
Change & $23.3 \pm 10.6$ & $20.3 \pm 5.6$ & $1.6 \pm 2.0$ & $1.5 \pm 4.8$ \\
\multicolumn{5}{c}{ Haemoglobin $(\mathrm{g} / \mathrm{L})$} \\
Baseline & $125.8 \pm 3.7$ & $133.7 \pm 2.0$ & $132.2 \pm 3.3$ & $126.8 \pm 1.9$ \\
Follow-up & $130.1 \pm 2.3$ & $136.3 \pm 4.2$ & $131.6 \pm 3.9$ & $129.0 \pm 4.6$ \\
Change & $4.3 \pm 4.0$ & $2.7 \pm 3.3$ & $-0.6 \pm 2.3$ & $2.2 \pm 3.6$ \\
& \multicolumn{5}{c}{ sTfR-index } \\
Baseline & $1.4 \pm 0.3$ & $1.2 \pm 0.3$ & $1.1 \pm 0.2$ & $0.7 \pm 0.6$ \\
Follow-up & $0.8 \pm 0.8$ & $0.7 \pm 0.1$ & $0.9 \pm 0.1$ & $0.6 \pm 0.9$ \\
Change & $-0.3 \pm 0.2$ & $-0.6 \pm 0.2$ & $-0.18 \pm 0.1$ & $-0.0 \pm 0.1$ \\
\hline
\end{tabular}

Note: $60 \mathrm{mg}$ and $80 \mathrm{mg}$ iron: ferrous sulfate, sTfR-Index: soluble transferrin receptor-ferritin index. Normal ranges for haematological markers: ferritin $>20 \mu \mathrm{g} / \mathrm{L}$; haemoglobin $115-165 \mathrm{~g} / \mathrm{L}$; soluble transferrin receptor $0.9-2.30 \mathrm{mg} / \mathrm{L}$.

\subsubsection{Baseline}

Kruskal-Wallis analyses performed on iron status markers for the three iron deficient groups (60 mg, $80 \mathrm{mg}$ and placebo) confirmed there were no significant between group differences in ferritin $(p=0.38)$, haemoglobin $(p=0.34)$ or sTfR-Index $(p=0.82)$ at baseline. As shown in Table 3 , analyses comparing iron sufficient (controls) and iron deficient participants (60 mg, $80 \mathrm{mg}$ and placebo groups combined) revealed that controls had significantly higher ferritin $(p<0.01)$ and lower sTfR-Index $(p<0.01)$ than the combined iron deficient groups at baseline, but no difference in haemoglobin $(p=0.30)$.

Table 3. Comparison of haematological markers at baseline, follow-up and change scores.

\begin{tabular}{|c|c|c|c|}
\hline Comparisons ( $p$ value) & Ferritin & Haemoglobin & sTfR-Index \\
\hline \multicolumn{4}{|c|}{ Baseline } \\
\hline Controls $v s$. Iron deficient & $<0.01$ & 0.30 & $<0.01$ \\
\hline \multicolumn{4}{|c|}{ Follow-up } \\
\hline Placebo vs. Controls, $60 \mathrm{mg}, 80 \mathrm{mg}$ & $<0.01$ & 1.0 & 0.11 \\
\hline \multicolumn{4}{|c|}{ Change score } \\
\hline Iron treatment $v s$. Placebo & $<0.01$ & 0.45 & 0.07 \\
\hline
\end{tabular}




\subsubsection{Follow-Up}

Analysis of iron status at follow-up revealed a significant difference between the placebo group $v s$. the $60 \mathrm{mg}, 80 \mathrm{mg}$ and iron sufficient controls combined in ferritin $(p \leq 0.01)$, but no difference in haemoglobin $(p=1.0)$ or sTfR-Index $(p=0.11)$ (as shown in Table 3$)$. Post hoc analysis showed the placebo group had significantly lower ferritin at follow-up than the $60 \mathrm{mg}$ iron group, $80 \mathrm{mg}$ iron group and controls $(p=0.02, p=0.02, p=0.04)$. There was no significant difference in ferritin between controls and $60 \mathrm{mg}$ iron $(p=0.57)$, controls and $80 \mathrm{mg}$ iron $(p=0.87)$, or $60 \mathrm{mg}$ and $80 \mathrm{mg}$ groups $(p=0.89)$ at follow-up.

\subsubsection{Change Scores}

Change scores between baseline and follow-up for the iron treatment (60 mg and $80 \mathrm{mg}$ combined) and placebo (iron deficient placebo and iron sufficient controls combined) groups were compared using one-way ANOVA. As shown in Table 3, the analyses revealed that the increase in ferritin levels was significantly greater following iron treatment compared with placebo, $F(1,23)=8.46, p \leq 0.01$. There were no differences in haemoglobin change, $F(1,22)=0.60, p=0.45$, or sTfR-Index change, $F(1,15)=3.95, p=0.07$, between iron treatment and placebo groups.

Based on our criteria for iron deficiency (ferritin $<20 \mu \mathrm{g} / \mathrm{L}$, haemoglobin 115-165 g/L, soluble transferrin receptor $0.9-2.30 \mathrm{mg} / \mathrm{L}$, A1GP $0.51-1.17 \mathrm{~g} / \mathrm{L})$, at follow-up six $(75 \%)$ iron deficient participants on $60 \mathrm{mg}$ iron became iron sufficient and one remained iron deficient (13\%). Four iron deficient $(57 \%)$ participants on $80 \mathrm{mg}$ iron became iron sufficient and two remained iron deficient (28\%). Two iron deficient (25\%) participants on placebo become iron sufficient and four (50\%) remained iron deficient. All except one (who became iron deficient) of the iron sufficient controls $(80 \%)$ remained iron sufficient (see Table 4$)$. Of the participants on iron treatment, there was no difference in ferritin change score between oral contraceptive pill users and non-users $(p=0.94)$.

Table 4. Iron status outcome, compliance, side effects and treatment guesses by treatment group.

\begin{tabular}{|c|c|c|c|c|c|c|c|c|c|c|c|c|}
\hline \multirow[b]{2}{*}{$\begin{array}{l}\text { Participant } \\
\text { group }\end{array}$} & \multicolumn{3}{|c|}{ Outcome } & \multirow[t]{2}{*}{ Compliance (\%) * } & \multicolumn{5}{|c|}{ Side effects } & \multicolumn{3}{|c|}{ Treatment gues } \\
\hline & $\mathscr{\Omega}$ & $\Theta$ & 嵏 & & $\bar{z}$ & $\begin{array}{l}\mathbb{J} \\
\text { 产 } \\
\text { Z }\end{array}$ & 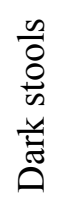 & 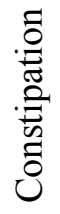 & 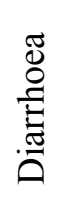 & రే & $\begin{array}{l}0 \\
\frac{8}{0} \\
\frac{\pi}{2} \\
2\end{array}$ & 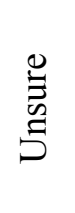 \\
\hline $60 \mathrm{mg}$ iron $(n=7)$ & 6 & 1 & 1 & $85.7 \pm 17.7$ & 5 & 1 & 1 & 0 & 0 & 6 & 1 & 0 \\
\hline $80 \mathrm{mg}$ iron $(n=6)$ & 4 & 2 & 2 & $93.3 \pm 10.6$ & 2 & 1 & 4 & 1 & 2 & 3 & 2 & 1 \\
\hline ID placebo $(n=5)$ & 1 & 4 & 3 & $92.3 \pm 5.3$ & 4 & 1 & 0 & 0 & 2 & 2 & 1 & 2 \\
\hline IS controls $(n=6)$ & 5 & 1 & 2 & $88.7 \pm 9.1$ & 5 & 1 & 1 & 0 & 0 & 1 & 5 & 0 \\
\hline
\end{tabular}

$60 \mathrm{mg}$ and $80 \mathrm{mg}$ iron: ferrous sulfate, ID: iron deficient; IS: iron sufficient; DNF: Did not finish;

* Compliance is based on the percentage of capsules participants returned at the end of the 16 weeks trial period out of a maximum of 112 . 


\subsection{Side Effects and Compliance}

Reported side effects included nausea, darkening of stools and constipation. While these were more commonly reported by participants in the $80 \mathrm{mg}$ elemental iron group, particularly dark stools (see Table 4), a Fischer's exact test indicated there was no statistically significant differences in the frequency of reported side effects between the $60 \mathrm{mg}$ and $80 \mathrm{mg}$ groups $(p=0.29)$, the placebo and controls $(p=0.55)$, or between the treatment and placebo groups $(p=0.42)$. Kruskal-Wallis analyses performed on compliance scores (\% of capsules taken) showed no statistically significant difference between the $60 \mathrm{mg}$ and $80 \mathrm{mg}$ groups $(p=0.22)$, between the placebo and controls $(p=0.25)$, or between the treatment and placebo groups $(p=0.60)$.

\subsection{Participants’ Treatment Guesses}

Of the 13 participants taking iron supplements who completed the trial, 9 (69\%) correctly guessed they were taking iron supplements. A Fischer's exact test showed no difference in the number of correct treatment guesses between the $60 \mathrm{mg}$ and $80 \mathrm{mg}$ groups $(p=0.27)$. Of the 11 participants taking placebo capsules who completed the trial, 6 participants $(56 \%)$ correctly guessed that they were taking placebo capsules. There was also no significant difference in the number of correct treatment guesses between the placebo and control groups $(p=0.08)$.

\section{Discussion}

\subsection{Change in Iron Status}

Limited knowledge exists on the efficacy of different doses of iron supplementation on iron status in non-pregnant young women with latent iron deficiency. Our study aimed to determine a ferrous sulfate dose that improves iron stores in women with latent iron deficiency, while minimising side effects. At follow-up, iron deficient participants who were randomised to ferrous sulfate (60 mg or $80 \mathrm{mg}$ ) had significant improvements in their ferritin from baseline levels. Following this improvement, there was no difference in ferritin when compared to controls at follow-up. Iron deficient participants randomised to placebo had significantly lower ferritin than iron treatment groups and controls at follow-up. This shows that 16 weeks of elemental iron is effective in normalising iron levels in most participants in this population of young women, and that without such iron treatment iron stores remain depleted. This pilot study had $68 \%$ power to detect a difference in ferritin change score between treatment or no treatment groups. The analysis showed a significantly higher ferritin change in treatment compared to no treatment groups. The power in this study will be used to inform the sample size in future studies as data on treatment of latent iron deficiency in non-pregnant women is limited.

Ferritin increased in iron deficient participants on placebo to a much lesser degree than those on iron treatment. Altering dietary iron was not part of the intervention and participants were not given any advice about changing their diet in order to keep their current intakes stable during the trial. It is possible that participants altered their dietary iron intake, which may explain some of the change in iron status and is a limitation that must be acknowledged. However, participants remained blinded to 
their iron status until the completion of the trial, so it is just as likely that iron sufficient controls changed their dietary iron intake as the iron deficient participants. Results also demonstrated that a daily $60 \mathrm{mg}$ dose was as effective as an $80 \mathrm{mg}$ dose in treating latent iron deficiency. Seventy five per cent of participants on $60 \mathrm{mg}$ iron dose became iron sufficient at the end of the trial as compared with $57 \%$ of participants in the $80 \mathrm{mg}$ iron group. However, there was no significant difference in iron status at follow-up between the $60 \mathrm{mg} v \mathrm{~s} .80 \mathrm{mg}$ iron groups.

A systematic review of the literature has actually shown that weekly dosing at $60-120 \mathrm{mg}$ is adequate for treating iron deficiency in menstruating women [11], however national guidelines recommend 80 or $105 \mathrm{mg}$ daily, which is also recommended by General Practitioners in Australia [10]. We have shown that $60 \mathrm{mg}$ daily is efficacious in young women with latent iron deficiency.

\subsection{Compliance}

The incidence of reported side effects was not statistically significantly different between placebo or treatment groups in this trial. The gastrointestinal effects of iron supplementation appear to be highly individual. Clear dose related side effects have been reported in previous studies using low (15 mg) and high doses $(222 \mathrm{mg})[8,15]$, whereas others have found no difference in side effects between placebo and treatment groups, even when daily doses of $260 \mathrm{mg}$ were used [27]. In the current study, there was no statistically significant difference in the compliance between groups. Galloway et al. (1994) reviewed literature on participants' compliance with iron supplement regimes in research studies and reported that compliance decreases when dose increases, however, as in the current study, Galloway found little evidence of side effects causing low compliance [28].

\subsection{Side Effects and Treatment Guess}

This study also aimed to examine the effect of potential side effects of the two different doses of iron supplementation on awareness of blinding to treatment groups. To assist with blinding, capsules were used in the study rather than tablets. This is due to ferrous sulfate being slightly green in colour and having a distinctly metallic taste. Therefore, to produce tablets for a blinded trial would involve finding inactive compounds to mimic or hide both the colour and taste of ferrous sulfate. Seventy seven per cent of participants in the treatment groups could guess that they were on iron, which is much higher than the $48 \%$ of 191 correctly guessing they were taking iron reported by Makrides et al. [16], though this study was in pregnant women who were obviously undergoing significant bodily changes making any additional effects of iron treatment difficult to identify. In the current study, the incidence of reported side effects was not different between treatment groups and placebo. This suggests that factors other than side effects play a role in the identification of their treatment, such as perhaps feeling more energetic. Although there was no formal assessment of fatigue and vitality in the current study, Patterson et al. (2001) showed improved vitality and decreased fatigue after treatment of iron deficiency in young women [4]. 


\subsection{Limitations}

Several limitations of this study must be acknowledged. These include the small sample size, and low power, which are likely to have affected the reliability of results. Some participants may have self-selected for this study given that they thought they were iron deficient, however, we made it clear that participants with iron deficiency within the 12 months prior to their enrolment in the study were not eligible. Also, physical activity and dietary intake were not assessed. These factors may have influenced individuals iron status at follow-up [29]. Despite possible influence of day-to-day variation [30], menstruation [31] and seasonal variation [32] on hematological results, the timing of the blood testing was not controlled to prevent unnecessary increased participant burden.

\section{Conclusions}

Results of this study revealed that a $60 \mathrm{mg}$ iron dose can normalize iron status in non-pregnant young women with latent iron deficiency. No differences were found in the incidence of reported side effects or the level of compliance between treatment groups and placebo. Further double-blinded trials should examine the effectiveness of iron doses lower than $60 \mathrm{mg}$ for improving iron status in young women, and to determine if awareness of treatment allocation is reduced.

\section{Acknowledgments}

This research was supported by Meat and Livestock Australia. Alecia J Leonard receives a University of Newcastle Scholarship and a top-up scholarship from Meat and Livestock Australia.

\section{Author Contributions}

All authors contributed to designing this research (project conception, development of overall research plan, and study oversight); AL conducted research (hands-on conduct of the experiments and data collection); $\mathrm{AL}$ analysed data or performed statistical analysis; AL was responsible for the first draft of the paper AP, CC and KC all had major contribution to editing; All authors had responsibility for final content.

\section{Conflicts of Interest}

The authors declare no conflict of interest.

\section{References}

1. World Health Organization. Iron deficiency anaemia: Assessment, prevention, and control. A guide for programme managers. Available online: http:/www.who.int/nutrition/publications/ en/ida_assessment_prevention_control.pdf(accessed on 10 August 2013).

2. Fayet, F.; Flood, V.; Petocz, P.; Samman, S. Relative and biomarker-based validity of a food frequency questionnaire that measures the intakes of vitamin $\mathrm{B}(12)$, folate, iron, and zinc in young women. Nutr. Res. 2010, 31, 14-20. 
3. Patterson, A.J.; Brown, W.J.; Powers, J.R.; Roberts, D.C. Iron deficiency, general health and fatigue: Results from the Australian Longitudinal Study on Women's Health. Qual. Life Res. 2000, 9, 491-497.

4. Patterson, A.J.; Brown, W.J.; Roberts, D.C. Dietary and supplement treatment of iron deficiency results in improvements in general health and fatigue in Australian women of childbearing age. J. Am. Coll. Nutr. 2001, 20, 337-342.

5. Mora, J.O. Iron supplementation: Overcoming technical and practical barriers. J. Nutr. 2002, 132, S853-S855.

6. International Nutritional Anemia Consultative Group (INACG). Guidelines for the Use of Iron Supplements to Prevent and Treat. Iron Deficiency Anaemia; Stoltzfus, R.J., Dreyfuss, M.L., Ed.; International Life Sciences Institute: Washington, DC, USA, 1998.

7. Goddard, A.F.; James, M.W.; McIntyre, A.S.; Scott, B.B.; British Soc, G. Guidelines for the management of iron deficiency anaemia. Gut 2011, 60, 1309-1316.

8. Australian Medicines Handbook Pty Ltd. Australian Medicines Handbook; Australian Medicines Handbook Pty Ltd.: Adelaide, Australia, 2010.

9. Therapeutic Guidelines Ltd. Therapeutic Guidelines: Gastrointestinal; eTG: Melbourne, Australia, 2006.

10. National Prescribing Service. Available online: http://www.nps.org.au/publications/healthprofessional/nps-news/2010/iron-anaemia (accessed on 10 September 2013).

11. Fernandez-Gaxiola, A.C.; De-Regil, L.M. Intermittent iron supplementation for reducing anaemia and its associated impairments in menstruating women. Cochrane Database Syst. Rev. 2011, doi:10.1002/14651858.CD009218.pub2.

12. Hallberg, L.; Ryttinge, L.; Solvell, L. Side-effects of oral iron therapy-A double blind study of different iron compounds in tablet form. Acta Med. Scand. 1966, 180, 3-21.

13. Zhu, A.; Kaneshiro, M.; Kaunitz, J.D. Evaluation and Treatment of Iron Deficiency Anemia: A Gastroenterological Perspective. Dig. Dis. Sci. 2010, 55, 548-559.

14. Macdougall, I.C. Strategies for iron supplementation: Oral vs. intravenous. Kidney Int. 1999, 55, S61-S66.

15. Rimon, E.; Kagansky, N.; Kagansky, M.; Mechnick, L.; Mashiah, T.; Namir, M.; Levy, S. Are we giving too much iron? Low-dose iron therapy is effective in octogenarians. Am. J. Med. 2005, 118, 1142-1147.

16. Makrides, M.; Crowther, C.A.; Gibson, R.A.; Gibson, R.S.; Skeaff, C.M. Efficacy and tolerability of low-dose iron supplements during pregnancy: A randomized controlled trial. Am. J. Clin. Nutr. 2003, 78, 145-153.

17. Mozaffari-Khosravi, H.; Noori-Shadkam, M.; Fatehi, F.; Naghiaee, Y. Once Weekly Low-dose Iron Supplementation Effectively Improved Iron Status in Adolescent Girls. Biol. Trace Elem. Res. 2010, 135, 22-30.

18. Rockey, D. Treatment of iron deficiency. Gastroenterology 2006, 130, 1367-1368.

19. Leonard, A.; Hutchesson, M.; Patterson, A.; Chalmers, K.; Collins, C. Recruitment and retention of young women into nutrition research studies: Practical considerations. Trials 2014, 15, 23.

20. Ahmed, F.; Coyne, T.; Dobson, A.; McClintock, C. Iron status among Australian adults: Findings of a population based study in Queensland, Australia. Asia Pac. J. Clin. Nut. 2008, 17, 40-47. 
21. Suominen, P.; Punnonen, K.; Rajamaki, A.; Majuri, R.; Hanninen, V.; Irjala, K. Automated immunoturbidimetric method for measuring serum transferrin receptor. Clin. Chem. 1999, 45, 1302-1305.

22. Leonard, A.J.; Patterson, A.J.; Collins, C.E.; Chalmers, K.A. Is soluble transferrin receptor a useful marker in early stage iron deficiency? e-SPEN J. 2013, 8, e210-e212.

23. Larsson, A.P.M.; Hansson, L.-O.; Basu, S.; Axelsson, O. Reference values for a1-acid glycoprotein, a1-antitrypsin, albumin, haptoglobin, C-reactive protein, IgA, IgG and IgM during pregnancy. Acta Obstet. Gynecol. 2008, 87, 1084-1088.

24. Koulaouzidis, A.; Said, E.; Cottier, R.; Saeed, A.A. Soluble transferrin receptors and iron deficiency, a step beyond ferritin. A systematic review. J. Gastrointest. Liver Dis. 2009, 18, 345-352.

25. Olivares, M.; Walter, T.; Cook, J.D.; Hertrampf, E.; Pizarro, F. Usefulness of serum transferrin receptor and serum ferritin in diagnosis of iron deficiency in infancy. Am. J. Clin. Nutr. 2000, 72, 1191-1195.

26. Saghaei, M. Random Allocation Software. 2004. Available online: http://mahmoodsaghaei. tripod.com/Softwares/randalloc.html\#Random Allocation Software (accessed on 20 September 2013).

27. Bruner, A.B.; Joffe, A.; Duggan, A.K.; Casella, J.F.; Brandt, J. Randomised study of cognitive effects of iron supplementation in non-anaemic iron-deficient adolescent girls. Lancet 1996, 348, 992-996.

28. Galloway, R.; McGuire, J. Determinants of compliance with iron supplementation: Supplies, side effects, or psychology? Soc. Sci. Med. 1994, 39, 381-390.

29. Beard, J.; Tobin, B. Iron status and exercise. Am. J. Clin. Nutr. 2000, 72, S594-S597.

30. Borel, M.J.; Smith, S.M.; Derr, J.; Beard, J.L. Day-to-day variation in iron-status indexes in healthy-men and women. Am. J. Clin. Nutr. 1991, 54, 729-735.

31. Kim, I.; Yetley, E.A.; Calvo, M.S. Variations in iron-status measures during the menstrual cycle. Am. J. Clin. Nutr. 1993, 58, 705-709.

32. Maes, M.; Bosmans, E.; Scharpe, S.; Hendriks, D.; Cooremans, W.; Neels, H.; DeMeyer, F.; Dhondt, P.; Peeters, D. Components of biological variation in serum soluble transferrin receptor: Relationships to serum iron, transferrin and ferritin concentrations, and immune and haematological variables. Scand. J. Clin. Lab. Investig. 1997, 57, 31-41.

(C) 2014 by the authors; licensee MDPI, Basel, Switzerland. This article is an open access article distributed under the terms and conditions of the Creative Commons Attribution license (http://creativecommons.org/licenses/by/3.0/). 